\title{
Translational approaches to investigation of solid tumor malignancies: shifting landscapes, smarter strategies and tactics for therapeutic design, improving patient outcomes
}

"It is said that if you know your enemies and know yourself, you will not be imperiled in a bundred battles; if you do not know your enemies but do know yourself, you will win one and lose one; if you do not know your enemies nor yourself, you will be imperiled in every single battle."-Sun-Tzu

The above quote, from military strategist Sun-Tzu's The Art of War, exemplifies the conundrum of translational oncology in the $21^{\text {st }}$ century. For the past decade, we have found ourselves at a crossroads in questioning our previously limited understanding of molecular and cellular biology as it relates to cancer. As a consequence, with the stark acceleration of new findings that have shifted the paradigms of how we treat cancer patients in the clinic, it is imperative that both cancer research scientists and clinicians alike come to a common understanding and meet on common ground when it comes to devising rational clinical trials that will effectively translate findings in a laboratory into meaningful new forms of therapy that will both prolong survival and also improve quality of life for millions of patients diagnosed with cancer.

It gives me great pleasure to introduce this special issue of Annals of Translational Medicine, which is intended to tackle this very issue. I am especially pleased and honored by the contributions from numerous influential thought leaders in solid tumor oncology from leading institutions, including the Memorial Sloan-Kettering Cancer Center (MSKCC), the University of California San Francisco (UCSF), the University of North Carolina (UNC) at Chapel Hill, the Royal Marsden Hospital in London, the University of Minnesota, the University of New Mexico, and Duke University. Articles from these leading researchers, taken together, effectively paint the picture on a wide canvas and shine a spotlight on critical issues facing the translational oncology community. These leading experts provide a state-of-the-art assessment of timely questions spanning cancer research, from bedside to clinic (and back), including but not limited to accurate molecular assessment of genomic profiles, predictive and prognostic biomarkers, determination of appropriate treatments based on economic profiles, and also the financial toxicity stemming from the recent new wave of effective but expensive cancer-targeting therapies in lung cancer, breast cancer, colorectal cancer, and prostate cancer.

Drs. Mondaca and Yaeger from MSKCC are at the forefront of identifying the role of prognostic and predictive biomarkers in colorectal cancer, and the implications of identifying these biomarkers on efficacy of treatment in this disease. This is especially pertinent over the last decade, as next generation sequencing of colorectal cancers has expanded significantly with the discovery of additional mutations and variants of KRAS and NRAS, as well as therapeutic implications of other potential driving mutations such as $B R A F$ and HER2. Of special significance is the fact that subclinical populations that lead to eventual drug resistance are present at the time of diagnosis, rather than purely evolving secondary to selective pressure during the treatment phase. Drs. Mondaca and Yaeger address this challenging issue in the accompanying article.

Likewise, Drs. Smyth, Khan, and Valeri from the Royal Marsden Hospital discuss similar challenges in identifying biomarkers that can more effectively predict response to therapy in cancers of the upper gastrointestinal tract. Their important work along with the work of colleagues in correlating biomarkers with outcomes from patients enrolled in the seminal Medical Research Council Adjuvant Gastric Infusional Chemotherapy (MAGIC) phase III trial—which set a new standard for treatment of patients with resectable gastroesophageal cancer using perioperative chemotherapy-is discussed in detail in their review. They use this platform to discuss how analysis of past trials can better inform new prospective translational clinical trials, identify relative radiologic biomarkers of response, and address the cutting-edge technique of using patient-derived organoid models as potential aids for drug screening at the individual patient level.

Along the same lines, in lung cancer, Drs. Zaman and Bivona from UCSF are at the forefront of connecting advances in genomics to identification of superior therapeutic approaches in order to personalize treatment to individual patients. They discuss the effect of oncogenic alterations on treatment decisions for both non-small cell and small cell lung cancer, and the impact of post-therapy resistance mechanisms on shifting the paradigm of how lung cancer patients are treated in the clinic in response to the ever-evolving landscape of lung tumors over time. Furthermore, in this burgeoning era of immunotherapy 
primarily with checkpoint inhibition, they also elegantly review effective strategies for deploying this class of therapeutic drugs in lung cancer in the context of both the biologic challenges of residual disease after treatment and tumor heterogeneity.

Effective clinical management based on genomic assays must rest upon a solid foundation of accuracy of those diagnostic assays. For that reason, the research article from Drs. Henzler and Nelson and team from the University of Minnesota is especially important, as it describes in-depth the inherent challenges in optimizing microfluidic-based next generation sequencing that is used to provide clinicians better understanding of the underlying molecular biology of each individual patient's tumors. Validation of this type and, in fact, all pathology-based assays is critical to ensuring best possible clinical decisionmaking. Their perspectives as molecular pathologists provide detailed insight into this key role in translational clinical oncology.

Drs. Kornberg, Chou, Feng, and Ryan also delve deeply into this topic, specifically addressing innovations and understanding molecular tumor subtypes of prostate cancer, and how these advances are better informing improvements in tactics in treating this disease. Specifically, these authors focus on recognizing the role of DNA damage repair mutations in the pathogenesis of prostate cancer and tailoring therapies in light of this information and a better understanding of resistance mechanisms. Such considerations continue to inform the multipronged and interdisciplinary approach to treating patients with prostate adenocarcinoma, in the context of concurrent advances using anti-androgens, immunotherapy, and chemotherapy as well.

Likewise, Drs. Rao, Wiggins, and Lauer from the University of New Mexico provide their research findings analyzing survival outcomes for advanced renal cell carcinoma (RCC) patients in the era of targeted therapies. The landscape and potential for improved survival changed significantly in the mid 2000's with the discovery of the efficacy of tyrosine kinase inhibitors (TKIs). Their examination of outcomes in nearly 15,000 patients treated during the pre- and post-TKI eras through examination of the U.S. national Surveillance, Epidemiology and End Results (SEER) database provide supportive evidence that there is a clear overall benefit to TKIs, even more so in patients with the clear cell histology subtype of RCC.

An oft-underlooked and understudied aspect of metastatic cancers is the biology and approaches to treatment of solid tumor brain metastasis. Dissemination of cancer across the barrier and into the central nervous system (CNS) is especially prevalent in breast cancer. Yet, presence of brain metastasis has long been a criterion for exclusion of many patients from clinical trials. Drs. Brosnan and Anders from UNC are at the leading edge of identifying modular subtypes and patterns of brain metastasis of breast cancer. Patterns of CNS metastasis have especially emerged in hormone-driven forms of the disease spurring brain metastasis. Here, they provide a very thorough and high-level examination of current understanding of the biology of breast cancer brain metastasis, and the potential for novel targeted and immunotherapeutic strategies to effectively treat this form of metastasis in addition to systemic disease. Their discussion provides a strong argument and hope that more effective treatments can in fact be designed for this patient population and that these patients can be better integrated prospectively into future clinical trials.

Ultimately, nearly all of the focus of translational oncology has been on the biologic efficacy and comparing these potential benefits to potential physical risks of these new therapeutic classes of drugs. However, initially lost in the conversation, but with increasing attention nationally and internationally, the fact that these drugs are quite expensive and significantly increase the financial costs of overall cancer care has gained overdue attention. Drs. Tran and Zafar are in a prime position of authority to address this growing concern, on behalf of the oncology community as well as the patient community, of this potential for "financial toxicity". The emotional and mental burden to patients, and their caregivers, of financial barriers to care cannot be underestimated. This article importantly highlights the potentially devastating effects stemming from the rising cost of targeted therapies, and the authors propose potential interventions to reduce drug costs.

The interplay and dynamics of the physician-patient relationship have been transformed irrevocably in recent years. This transformation of the landscape has been forever altered—in fact, greatly improved—due to the steep rise in means to access information via the internet, and amplified even further by the rise of social media platforms. In this information age, patients no longer need to wait to be informed, but rather proactively acquire information even before first meeting their oncologists and care teams. It may be surprising to many researchers as well as physicians that patients also are asking to know more details about the science and the evidence-based medicine supporting — or not supporting - many of the decisions that are made for their care in the clinic. Empowerment of the modern cancer patient is playing a more important role than ever in the patient-care team relationship. For that reason, it gives me extreme pleasure to include in this issue a special article on the patient perspective from three leading cancer patient advocates-AnneMarie Ciccarella, Alicia Staley, and Dr. Aime Franco- 
so that those of us approaching cancer from the scientific side can gain a better appreciation from those who approach the problem of cancer from a more personal side.

All of the articles included in the special issue, regardless of specific site of disease of interest, carry a unifying theme that the revolution in translational solid tumor oncology is upon us, and that a seamless transition of the understanding between the laboratory and clinic is more essential than ever. Returning to this theme of strategies and tactics, and applying them judiciously based on current state of knowledge that is ever evolving, we will not be able to achieve our full potential for attacking cancer and its root cause and overcoming challenges of therapeutic drug resistance until we fully galvanize and synthesize our knowledge in the most deliberate and rational approach possible. In my accompanying editorial in this issue, I cite principles of military strategies and tactics from Sun-Tzu's tome and apply them to specific examples in which we can apply these ideas to translational oncology to maximize the impact of cancer treatments. Ultimately, the goal of cancer research is not to destroy, but to greatly improve, the human condition by most intelligently applying our knowledge to the maximum benefit of all patients.

\section{Acknowledgements}

I would like to thank all of the authors for the tremendous and valued contributions to the special issue, the journal's editorial team for bringing this special issue to fruition, and also to you, the readers, for sharing in our collective perspectives for the continued advancement of cancer research worldwide.

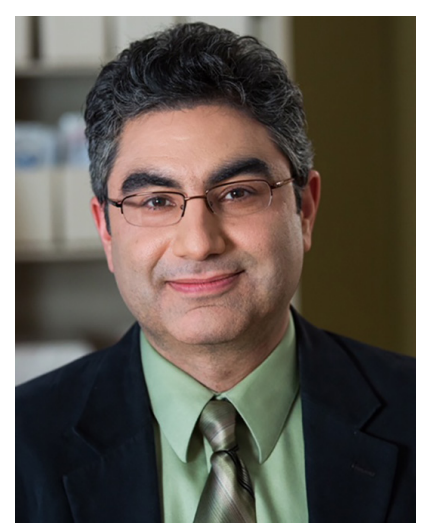

Emil Lou

Emil Lou, MD, PhD, FACP

Department of Medicine, Division of Hematology, Oncology and Transplantation, University of Minnesota, Minneapolis, Minnesota 55455, USA. (Email: emil-lou@umn.edu) doi: $10.21037 / \mathrm{atm} .2018 .04 .45$

Conflicts of Interest: The author has no conflicts of interest to declare. View this article at: http://dx.doi.org/10.21037/atm.2018.04.45

Cite this article as: Lou E. Translational approaches to investigation of solid tumor malignancies: shifting landscapes, smarter strategies and tactics for therapeutic design, improving patient outcomes. Ann Transl Med 2018;6(9):158. doi: 10.21037/ atm.2018.04.45 